\title{
Interplay of curvature and temperature in the Casimir-Polder interaction
}

\author{
Giuseppe Bimonte $\mathrm{B}^{1,2}$ and Thorsten Emig $^{3,4}$ \\ ${ }^{1}$ Dipartimento di Scienze Fisiche, Università di Napoli Federico II, \\ Complesso Universitario di Monte S. Angelo, Via Cintia, I-80126 Napoli, Italy \\ 2 INFN Sezione di Napoli, I-80126 Napoli, Italy \\ 3 Massachusetts Institute of Technology, MultiScale Materials Science for Energy and Environment, \\ Joint MIT-CNRS Laboratory (UMI 3466), Cambridge, Massachusetts 02139, USA \\ ${ }^{4}$ Massachusetts Institute of Technology, Department of Physics, Cambridge, Massachusetts 02139, USA
}

(Dated: April 24, 2022)

\begin{abstract}
We study the Casimir-Polder interaction at finite temperatures between a polarizable small, anisotropic particle and a non-planar surface using a derivative expansion. We obtain the leading and the next-to-leading curvature corrections to the interaction for low and high temperatures. Explicit results are provided for the retarded limit in the presence of a perfectly conducting surface.
\end{abstract}

PACS numbers: $12.20 .-\mathrm{m}, 03.70 .+\mathrm{k}, 42.25 . \mathrm{Fx}$

Keywords:

\section{INTRODUCTION}

Dispersion forces, i.e., long-range forces between polarizable bodies originating from quantum and thermal fluctuations of the electromagnetic (em) field, still constitute a very active field of research thanks to modern experimental techniques, which allow to observe these forces with unprecedented precision. Despite the similarity of the underlying physical mechanisms, dispersion forces are customarily denoted by different names (van der Waals, London, Casimir-Polder), depending on the size of the involved bodies and the separation scales. For reviews on dispersion forces, see 4 6]. In what follows we shall use the abbreviations $\mathrm{PP}$ and $\mathrm{PS}$ to denote in general the dispersion interaction of a small anisotropic particle (including atoms and molecules), with another particle or with a surface, respectively, while the abbreviation SS shall denote the interaction among two surfaces.

Among the three above manifestations of dispersion forces, PP forces are by far the simplest, because at separations that are large compared to their sizes, small dielectric particles can be modelled as simple dipoles. The situation gets much more complicated however when one or two macroscopic surfaces are involved, as it happens in the PS and in the SS cases respectivley, for then the characteristic many-body nature of dispersion forces entailed by their long-range character makes it very difficult to compute the interaction as soon as one (or two) non-planar surfaces are involved. Recent advances in Micro and Nano-Electro-Mechanical Systems prompted a strong interest in the study of geometry effects on dispersion forces. In the PS case, which is the specific object of this paper, geometry effects are important for devices used to trap atoms or molecules close a surface. For a review of recent experiments involving the $\mathrm{CP}$ interaction of atoms with microstructured surfaces, see [7-10].

Due to the difficulty of the problem, theoretical investigations of the PS interaction for non planar surfaces have been carried out only for a few specific geometries. The example of a uniaxially corrugated surface was studied numerically in Ref. 11 within a toy scalar field theory, while a rectangular dielectric gratings were considered in Ref. [12. In Refs. [13, 14] analytical results were obtained for the case of a perfectly conducting cylinder. A perturbative approach is presented in [15], where surfaces with smooth corrugations of any shape, but with small amplitude, were studied. The validity of the latter is restricted to particle-surface separations that are much larger than the corrugation amplitude. An alternative approach that becomes exact in the opposite limit of small particle-surface distances, was recently proposed in [17]. It is based on a systematic derivative expansion of the PS potential, that generalizes an analogous expansion which was successfully used [18, 20 to study the Casimir interaction between two non-planar surfaces. It has also been applied to other problems involving short range interactions between surfaces, like radiative heat transfer 21] and stray electrostatic forces between conductors [22]. From this expansion it was possible to obtain the leading and the next-to-leading curvature corrections to the $\mathrm{PS}$ interaction.

In current $\mathrm{CP}$ experiments, it is often of interest to understand how the atom-surface interaction is modified by the temperature of the surface and/or of the environment. The temperature dependence of the $\mathrm{CP}$ interaction has been first demonstrated in the experiment 23. involving a magnetically trapped ${ }^{87} \mathrm{Rb}$ Bose-Einstein condensate placed at a distance of a few microns from a heated fused silica substrate. Another very recent experiment 24 probed the thermal CP potential between $\mathrm{Cs}^{*}\left(7 \mathrm{D}_{3 / 2}\right)$ atoms and a hot sapphire substrate at a distance around $100 \mathrm{~nm}$, in the temperature range from 500 to $1,000 \mathrm{~K}$, paving the way to the control of atom-surface interactions by thermal fields. Temperature corrections to the PS potential for planar surfaces have been studied before by several authors 2539 , both at equilibrium and out of equilibrium. For non-planar surfaces, the only study known to us is [4], where the thermal PS potential for a particle near a microsphere has been studied using a multipole expansion. In this paper we use the 


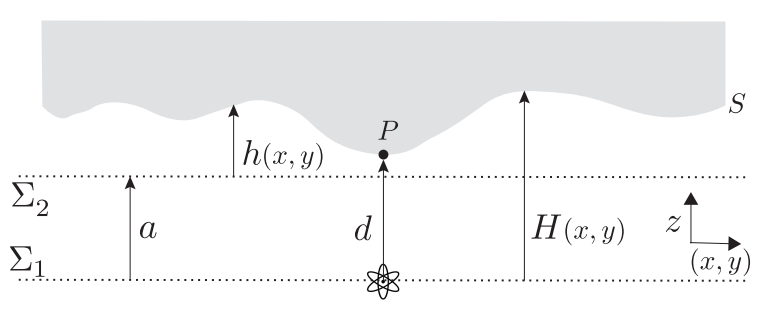

FIG. 1: Coordinates parametrizing a configuration consisting of a small particle near a gently curved surface.

derivative expansion developed in [17] to study the interplay of curvature and temperature in the PS potential of an anisotropic small particle in thermal equilibrium with a gently curved surface of any shape. For simplicity we consider here the idealized case of a perfectly conducting surface, and we postpone the study of dielectric surfaces to a future work.

The paper is organized as follows. In Sec. II we derive the general structure of the derivative expansion at finite temperature for an arbitrary material surface, and simplify the results for a perfectly conducting surface. In Sec. III curvature corrections for the retarded CasimirPolder interaction are computed for an anisotropic particle in a small temperature expansion and in the classical high temperature limit. Implications and possible extensions of our results are discussed in Sec. IV.

\section{DERIVATIVE EXPANSION OF THE PARTICLE-SURFACE POTENTIAL}

We consider a particle (an 'atom,' a molecule, or any polarizable micro-particle) that is in thermal equilibrium with a dielectric surface $S$ at temperature $T$. We assume that the particle is small enough (compared to the scale of its separation $d$ to the surface), such that its response to em fields is fully described by the dynamic electric dipolar polarizability tensor $\alpha_{\mu \nu}(\omega)$. (We assume for simplicity that the particle has a negligible magnetic polarizability, as is usually the case). Let $\Sigma_{1}$ be the plane through the particle which is orthogonal to the distance vector (which we take to be the $\hat{\mathbf{z}}$ axis) connecting the particle to the point $P$ of $S$ closest to the particle. We assume that the surface $S$ is characterized by a smooth profile $z=H(\mathbf{x})$, where $\mathbf{x}=(x, y)$ is the vector spanning $\Sigma_{1}$, with origin at the particle's position (see Fig. 1). In what follows greek indices $\mu, \nu, \ldots$ label all coordinates $(x, y, z)$, while latin indices $i, j, k \ldots$ refer to $(x, y)$ coordinates in the plane $\Sigma_{1}$. Throughout we adopt the convention that repeated indices are summed over.

The exact interaction potential at finite temperature $T$ is given by the scattering formula [41, 42]

$$
U=-k_{B} T \sum_{n=0}^{\prime} \operatorname{Tr}\left[\mathbb{T}^{(S)} \mathbb{U} \mathbb{T}^{(A)} \mathbb{U}\right]\left(\kappa_{n}\right) .
$$

Here $\mathbb{T}^{(S)}$ and $\mathbb{T}^{(A)}$ denote, respectively, the T-operators of the plate $S$ and the particle, evaluated at the Matsubara wave numbers $\kappa_{n}=2 \pi n k_{B} T /(\hbar c)$, and the primed sum indicates that the $n=0$ term carries weight $1 / 2$. In a plane-wave basis $|\mathbf{k}, Q\rangle$ [4] where $\mathbf{k}$ is the in-plane wave-vector, and $Q=E, M$ denotes respectively electric (transverse magnetic) and magnetic (transverse electric) modes, the translation operator $\mathbb{U}$ in Eq. (1) is diagonal with matrix elements $e^{-d q}$ where $q=\sqrt{k^{2}+\kappa_{n}^{2}} \equiv q(k)$, $k=|\mathbf{k}|$. The matrix elements of the T-operator for the small particle in the dipole approximation are

$$
\mathcal{T}_{Q Q^{\prime}}^{(A)}\left(\mathbf{k}, \mathbf{k}^{\prime}\right)=-\frac{2 \pi \kappa_{n}^{2}}{\sqrt{q q^{\prime}}} e_{Q \mu}^{(+)}(\mathbf{k}) \alpha_{\mu \nu}\left(\mathrm{i} c \kappa_{n}\right) e_{Q^{\prime} \nu}^{(-)}\left(\mathbf{k}^{\prime}\right)
$$

where $q^{\prime}=q\left(k^{\prime}\right), \mathbf{e}_{M}^{( \pm)}(\mathbf{k})=\hat{\mathbf{z}} \times \hat{\mathbf{k}}$ and $\mathbf{e}_{E}^{( \pm)}(\mathbf{k})=$ $-1 / \kappa_{n}(\mathrm{i} k \hat{\mathbf{z}} \pm q \hat{\mathbf{k}})$, with $\hat{\mathbf{k}}=\mathbf{k} / k$. There are no analytical formulae for the elements of the T-operator of a curved plate $\mathbb{T}^{(S)}$, and its computation is in general quite challenging, even numerically. However, it has been shown in Ref. 17 that at $T=0$ and in the classical limit for any smooth surface it is possible to compute the leading curvature corrections to the potential in the experimentally relevant limit of small separations. The key idea is that the PS interaction falls off rapidly with separation, and it is thus reasonable to expect that the potential $U$ is mainly determined by the geometry of the surface $S$ in a small neighborhood of the point $P$ of $S$ which is closest to the particle. This physically plausible idea suggests that for small separations $d$ the potential $U$ can be expanded as a series expansion in an increasing number of derivatives of the height profile $H$, evaluated at the particles's position. Here we extend this idea to compute thermal corrections to the potential at small separations. Up to fourth order, and assuming that the surface is homogeneous and isotropic, the most general expression which is invariant under rotations of the $(x, y)$ coordinates, and that involves at most four derivatives of $H$ (but no first derivatives since $\nabla H(\mathbf{0})=0$ ) can be expressed (up to $\left.\mathcal{O}\left(d^{-1}\right)\right)$ in the form

$$
\begin{gathered}
U=-\frac{k_{B} T}{d^{3}} \sum_{n=0}^{\infty}\left\{\beta_{1}^{(0)} \alpha_{\perp}+\beta_{2}^{(0)} \alpha_{z z}+d \times\left[\left(\beta_{1}^{(2)} \alpha_{\perp}+\beta_{2}^{(2)} \alpha_{z z}\right) \nabla^{2} H+\beta_{3}^{(2)}\left(\partial_{i} \partial_{j} H-\frac{1}{2} \nabla^{2} H \delta_{i j}\right) \alpha_{i j}\right]+d^{2} \times\right. \\
\left.\left[\beta^{(3)} \alpha_{z i} \partial_{i} \nabla^{2} H+\left(\nabla^{2} H\right)^{2}\left(\beta_{1}^{(4)} \alpha_{\perp}+\beta_{2}^{(4)} \alpha_{z z}\right)+\left(\partial_{i} \partial_{j} H\right)^{2}\left(\beta_{3}^{(4)} \alpha_{\perp}+\beta_{4}^{(4)} \alpha_{z z}\right)+\beta_{5}^{(4)} \nabla^{2} H\left(\partial_{i} \partial_{j} H-\frac{1}{2} \nabla^{2} H \delta_{i j}\right) \alpha_{i j}\right]\right\}
\end{gathered}
$$




\begin{tabular}{|l|l|l|l|}
\hline $\mathrm{p}$ & $\mathrm{q}$ & $\times e^{-2 \xi}$ & $\times \operatorname{Ei}(2 \xi)$ \\
\hline 0 & 1 & $\frac{1}{8}\left(1+2 \xi+4 \xi^{2}\right)$ & 0 \\
& 2 & $\frac{1}{4}(1+2 \xi)$ & 0 \\
2 & 1 & $-\frac{1}{32}\left(3+6 \xi+6 \xi^{2}+4 \xi^{3}\right)$ & $-\frac{\xi^{4}}{4}$ \\
& 2 & $-\frac{1}{16}\left(1+2 \xi-2 \xi^{2}+4 \xi^{3}\right)$ & $\xi^{2}\left(1-\frac{\xi^{2}}{2}\right)$ \\
3 & 3 & $-\frac{1}{32}\left(3+6 \xi+2 \xi^{2}-4 \xi^{3}\right)$ & $\frac{\xi^{4}}{4}$ \\
3 & & $\frac{1}{32}\left(1+2 \xi-2 \xi^{2}+4 \xi^{3}\right)$ & $-\frac{\xi^{2}}{4}\left(2-\xi^{2}\right)$ \\
4 & 1 & $\frac{1}{384}\left(3+6 \xi+15 \xi^{2}+22 \xi^{3}+2 \xi^{4}-4 \xi^{5}\right)$ & $\frac{\xi^{4}}{48}\left(6-\xi^{2}\right)$ \\
& 2 & $-\frac{1}{960}\left(15+542 \xi+259 \xi^{2}-546 \xi^{3}-14 \xi^{4}+28 \xi^{5}\right)$ & $-\xi^{2}\left(2-\frac{7 \xi^{2}}{6}+\frac{7 \xi^{4}}{120}\right)$ \\
3 & 3 & $\frac{1}{192}\left(15+30 \xi-9 \xi^{2}+70 \xi^{3}+2 \xi^{4}-4 \xi^{5}\right)$ & $\frac{\xi^{4}}{24}\left(18-\xi^{2}\right)$ \\
& 4 & $\frac{1}{480}\left(45+218 \xi-59 \xi^{2}+146 \xi^{3}+14 \xi^{4}-28 \xi^{5}\right)$ & $\frac{\xi^{4}}{60}\left(40-7 \xi^{2}\right)$ \\
& 5 & $\frac{1}{96}\left(9+18 \xi-27 \xi^{2}+50 \xi^{3}-2 \xi^{4}+4 \xi^{5}\right)$ & $\xi^{4}\left(1+\frac{\xi^{2}}{12}\right)$ \\
\hline
\end{tabular}

TABLE I: The coefficients $\beta_{q}^{(p)}(\xi)$ are obtained by multiplying the third column by $e^{-2 \xi}$, and adding the fourth column times $\operatorname{Ei}(2 \xi)=-\int_{2 \xi}^{\infty} d t \exp (-t) / t$

where $\alpha_{\perp}=\alpha_{x x}+\alpha_{y y}$, and it is understood that all derivatives of $H(\mathbf{x})$ are evaluated at the atom's position, i.e., for $\mathbf{x}=\mathbf{0}$. The Matsubara sum runs now over the rescaled wave numbers $\xi_{n}=\kappa_{n} d$ at which the dimensionless coefficient functions $\beta_{p}^{(p)}(\xi)$ are evaluated. For a general dielectric surface these functions depend also on any other dimensionless ratio of frequencies characterizing the material of the surface. The derivative expansion in Eq. (3) can be formally obtained by a re-summation of the perturbative series for the potential for small in-plane momenta $\mathbf{k}$ 17. We note that there are additional terms involving four derivatives of $H$ which, however, yield contributions $\sim 1 / d$ (as do terms involving five derivatives of $H)$ and are hence neglected.

The coefficients $\beta_{q}^{(p)}$ in Eq. 33) can be extracted from the perturbative series of the potential $U$, carried to second order in the deformation $h(\mathbf{x})$, which in turn involves an expansion of the T-operator of the surface $S$ to the same order. The latter expansion was worked out in Ref. [43] for a dielectric surface characterized by a frequency dependent permittvity $\epsilon(\omega)$. It reads

$$
\begin{aligned}
& \mathcal{T}_{Q Q^{\prime}}^{(S)}\left(\mathbf{k}, \mathbf{k}^{\prime}\right)=(2 \pi)^{2} \delta^{(2)}\left(\mathbf{k}-\mathbf{k}^{\prime}\right) \delta_{Q Q^{\prime}} r_{Q}^{(S)}\left(i c \kappa_{n}, \mathbf{k}\right) \\
& +\sqrt{q q^{\prime}}\left[-2 B_{Q Q^{\prime}}\left(\mathbf{k}, \mathbf{k}^{\prime}\right) \tilde{h}\left(\mathbf{k}-\mathbf{k}^{\prime}\right)\right. \\
& \left.+\int \frac{d^{2} \mathbf{k}^{\prime \prime}}{(2 \pi)^{2}}\left(B_{2}\right)_{Q Q^{\prime}}\left(\mathbf{k}, \mathbf{k}^{\prime} ; \mathbf{k}^{\prime \prime}\right) \tilde{h}\left(\mathbf{k}-\mathbf{k}^{\prime \prime}\right) \tilde{h}\left(\mathbf{k}^{\prime \prime}-\mathbf{k}^{\prime}\right)+\ldots\right]
\end{aligned}
$$

where $\sigma_{3}=\operatorname{diag}(1,-1)$. For perfect conductors the coefficients $\beta_{q}^{(p)}$ are functions of $\xi$ only, and we list them in Table I]

The potential in Eq. (3) can be expressed also in the radii of curvature, $R_{1}$ and $R_{2}$, of the surface $S$ at $P$. The 
local expansion of $H$ can be chosen as $H=d+x^{2} /\left(2 R_{1}\right)+y^{2} /\left(2 R_{2}\right)+\cdots$ so that the derivative expansion of $U$ reads

$$
\begin{aligned}
U & =-\frac{k_{B} T}{d^{3}} \sum_{n=0}^{\infty}\left\{\beta_{1}^{(0)} \alpha_{\perp}+\beta_{2}^{(0)} \alpha_{z z}+\left(\frac{d}{R_{1}}+\frac{d}{R_{2}}\right)\left(\beta_{1}^{(2)} \alpha_{\perp}+\beta_{2}^{(2)} \alpha_{z z}\right)+\frac{\beta_{3}^{(2)}}{2}\left(\frac{d}{R_{1}}-\frac{d}{R_{2}}\right)\left(\alpha_{x x}-\alpha_{y y}\right)\right. \\
& +d^{2} \beta^{(3)} \alpha_{z i} \partial_{i}\left(\frac{1}{R_{1}}+\frac{1}{R_{2}}\right)+\left(\frac{d}{R_{1}}+\frac{d}{R_{2}}\right)^{2}\left(\beta_{1}^{(4)} \alpha_{\perp}+\beta_{2}^{(4)} \alpha_{z z}\right) \\
& \left.+\left[\left(\frac{d}{R_{1}}\right)^{2}+\left(\frac{d}{R_{2}}\right)^{2}\right]\left(\beta_{3}^{(4)} \alpha_{\perp}+\beta_{4}^{(4)} \alpha_{z z}\right)+\frac{\beta_{5}^{(4)}}{2}\left[\left(\frac{d}{R_{1}}\right)^{2}-\left(\frac{d}{R_{2}}\right)^{2}\right]\left(\alpha_{x x}-\alpha_{y y}\right)\right\},
\end{aligned}
$$

where the dependence of the coefficients $\beta_{q}^{(p)}(\xi)$ on $\xi_{n}$ is again not shown explicitly.

\section{RETARDED PARTICLE-SURFACE POTENTIAL}

The $\beta$ coefficients in Eq. (3) are significantly different from zero only for rescaled frequencies $\xi_{n} \lesssim 1$. Therefore, for separations small compared to the radii of surface curvature but $d \gg c / \omega_{r}$, where $\omega_{r}$ is a typical resonance frequency of the particle (atom), we can replace $\alpha_{\mu \nu}(\mathrm{i} c \kappa)$ in Eqs. (37) by its static limit $\alpha_{\mu \nu}(0) \equiv \alpha_{\mu \nu}^{0}$. Then a small temperature expansion of the potential can be obtained by evaluating the sum using the Abel-Plana formula. This yields for $d \ll \lambda_{T}=\hbar c /\left(2 \pi k_{B} T\right)$ the retarded Casimir-Polder potential

$U_{\mathrm{CP}}=-\frac{\hbar c}{\pi d^{4}}\left[\alpha_{\perp}^{0} \eta_{\perp}+\alpha_{z z}^{0} \eta_{z z}+\alpha_{z i}^{0} \eta_{z i}+\left(\alpha_{x x}^{0}-\alpha_{y y}^{0}\right) \eta_{x y}\right]$

We performed the temperature expansion up to order $\left(d / \lambda_{T}\right)^{5}$. This order is the smallest one for which thermal corrections become visible in all terms of the following coefficients that describe curvature corrections up to order $\left(d / R_{i}\right)^{2}$,

$$
\begin{aligned}
\eta_{\perp} & =\frac{1}{8}-\frac{1}{360}\left(\frac{d}{\lambda_{T}}\right)^{4}-\left(\frac{d}{R_{1}}+\frac{d}{R_{2}}\right)\left[\frac{3}{40}-\frac{3 \zeta(5)}{32 \pi^{4}}\left(\frac{d}{\lambda_{T}}\right)^{5}\right]+\left(\frac{d}{R_{1}}+\frac{d}{R_{2}}\right)^{2}\left[\frac{3}{280}-\frac{3 \zeta(5)}{64 \pi^{4}}\left(\frac{d}{\lambda_{T}}\right)^{5}\right] \\
& +\left[\left(\frac{d}{R_{1}}\right)^{2}+\left(\frac{d}{R_{2}}\right)^{2}\right]\left[\frac{13}{280}+\frac{1}{360}\left(\frac{d}{\lambda_{T}}\right)^{4}\right] \\
\eta_{z z} & =\frac{1}{8}+\frac{1}{360}\left(\frac{d}{\lambda_{T}}\right)^{4}-\left(\frac{d}{R_{1}}+\frac{d}{R_{2}}\right)\left[\frac{1}{15}-\frac{\zeta(3)}{8 \pi^{2}}\left(\frac{d}{\lambda_{T}}\right)^{3}+\frac{1}{90}\left(\frac{d}{\lambda_{T}}\right)^{4}-\frac{3 \zeta(5)}{16 \pi^{4}}\left(\frac{d}{\lambda_{T}}\right)^{5}\right] \\
& -\left(\frac{d}{R_{1}}+\frac{d}{R_{2}}\right)^{2}\left[\frac{1}{240}-\frac{1}{45}\left(\frac{d}{\lambda_{T}}\right)^{2}+\frac{\zeta(3)}{4 \pi^{2}}\left(\frac{d}{\lambda_{T}}\right)^{3}-\frac{1}{60}\left(\frac{d}{\lambda_{T}}\right)^{4}+\frac{7 \zeta(5)}{16 \pi^{4}}\left(\frac{d}{\lambda_{T}}\right)^{5}\right] \\
& +\left[\left(\frac{d}{R_{1}}\right)^{2}+\left(\frac{d}{R_{2}}\right)^{2}\right]\left[\frac{3}{40}-\frac{1}{90}\left(\frac{d}{\lambda_{T}}\right)^{2}+\frac{1}{180}\left(\frac{d}{\lambda_{T}}\right)^{4}-\frac{\zeta(5)}{4 \pi^{4}}\left(\frac{d}{\lambda_{T}}\right)^{5}\right] \\
\eta_{z i} & =d^{2} \partial_{i}\left(\frac{1}{R_{1}}+\frac{1}{R_{2}}\right)^{5}\left[\frac{1}{30}-\frac{\zeta(3)}{16 \pi^{2}}\left(\frac{d}{\lambda_{T}}\right)^{3}+\frac{1}{180}\left(\frac{d}{\lambda_{T}}\right)^{4}-\frac{3 \zeta(5)}{32 \pi^{4}}\left(\frac{d}{\lambda_{T}}\right)^{5}\right] \\
\eta_{x y} & =-\left(\frac{d}{R_{1}}-\frac{d}{R_{2}}\right)\left[\frac{1}{40}+\frac{3 \zeta(5)}{64 \pi^{4}}\left(\frac{d}{\lambda_{T}}\right)^{5}\right]+\left[\left(\frac{d}{R_{1}}\right)^{2}-\left(\frac{d}{R_{2}}\right)^{2}\right]\left[\frac{9}{560}+\frac{1}{360}\left(\frac{d}{\lambda_{T}}\right)^{4}-\frac{3 \zeta(5)}{16 \pi^{4}}\left(\frac{d}{\lambda_{T}}\right)^{5}\right] .
\end{aligned}
$$

In the special case of a spherical atom near a cylindrical metallic shell at $T=0$, the above formula is in full agreement with Eq. (30) of Ref. [13, as well as with Eqs. (25)(26) and Eqs. (38)-(39) or Ref. 14]. The magnitude of the thermal corrections depends on the component of the polarizability tensor and the order of the curvature correction. The thermal contributions proportional to $\alpha_{z z}^{0}$ scales as $T^{3}$ at order $1 / R_{i}$ and as $T^{2}$ at order $1 / R_{i}^{2}$. The thermal terms proportional to lateral $(x, y)$ components of $\alpha^{0}$ are each reduced by $T^{2}$ compared those of $\alpha_{z z}^{0}$. 
This constitutes a manifestation of correlations between curvature and thermal effects in fluctuation induced interactions.

For completeness, we consider also the classical high temperature limit, where the Casimir free energy is given by the first term of the Matsubara sum in Eq. (1) only. From the limit $\kappa \rightarrow 0$ of the coefficients $\beta_{q}^{(p)}$ we obtain the classical free energy to order $\left(d / R_{i}\right)^{2}$ as

$$
\begin{gathered}
U_{\text {classical }}=-\frac{k_{B} T}{2} \frac{1}{d^{3}}\left\{\frac{1}{8} \alpha_{\perp}^{0}+\frac{1}{4} \alpha_{z z}^{0}-\frac{3}{64}\left(3 \frac{d}{R_{1}}+\frac{d}{R_{2}}\right) \alpha_{x x}^{0}-\frac{3}{64}\left(\frac{d}{R_{1}}+3 \frac{d}{R_{2}}\right) \alpha_{y y}^{0}-\frac{1}{16}\left(\frac{d}{R_{1}}+\frac{d}{R_{2}}\right) \alpha_{z z}^{0} \quad(13)\right. \\
\left.+\frac{1}{128}\left(17 \frac{d^{2}}{R_{1}^{2}}+5 \frac{d^{2}}{R_{2}^{2}}+2 \frac{d^{2}}{R_{1} R_{2}}\right) \alpha_{x x}^{0}+\frac{1}{128}\left(17 \frac{d^{2}}{R_{2}^{2}}+5 \frac{d^{2}}{R_{1}^{2}}+2 \frac{d^{2}}{R_{1} R_{2}}\right) \alpha_{y y}^{0}+\frac{1}{64}\left(5 \frac{d^{2}}{R_{1}^{2}}+5 \frac{d^{2}}{R_{2}^{2}}-2 \frac{d^{2}}{R_{1} R_{2}}\right) \alpha_{z z}^{0}\right\}
\end{gathered}
$$

The corrections to this limit due to higher order Matsubara terms decay exponentially in $d / \lambda_{T} \sim T$.

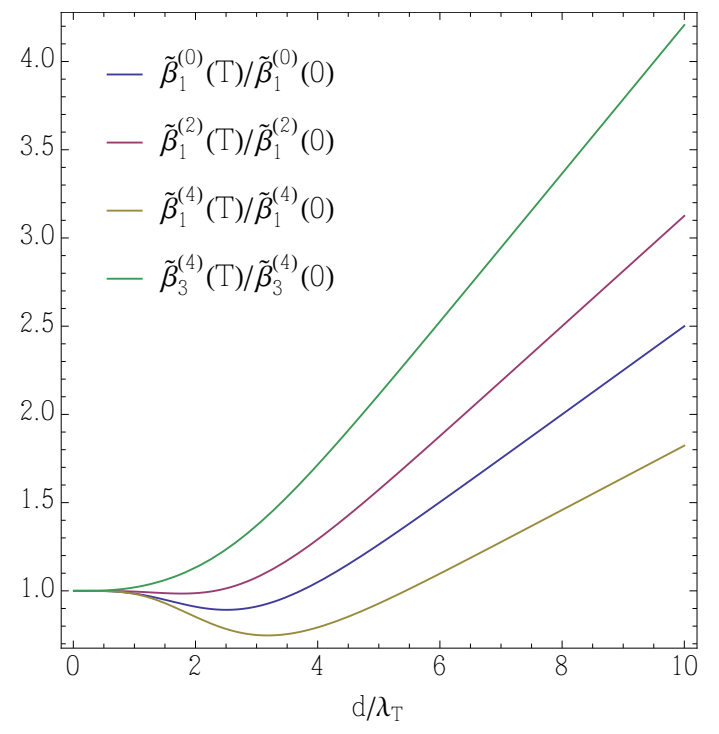

FIG. 2: Temperature dependence of the coefficients $\tilde{\beta}_{q}^{(p)}(T)=\left(d / \lambda_{T}\right) \sum_{n=0}^{\prime \infty} \beta_{q}^{(p)}\left(n d / \lambda_{T}\right)$ that mutliply $\alpha_{\perp}^{0}$ in Eq. (7), normalized to $\tilde{\beta}_{q}^{(p)}(T=0)=\int_{0}^{\infty} \beta_{q}^{(p)}(\xi) d \xi$.

It is instructive to study the full temperature dependence of the curvature corrections for $U$ and how it compares to $T$-dependence of the curvature independent terms. To this end we compute numerically the Matsubara sums $\tilde{\beta}_{q}^{(p)}(T)=\left(d / \lambda_{T}\right) \sum_{n=0}^{\prime \infty} \beta_{q}^{(p)}\left(n d / \lambda_{T}\right)$ and plot them normalized to their zero temperature limit $\tilde{\beta}_{q}^{(p)}(T=0)=\int_{0}^{\infty} \beta_{q}^{(p)}(\xi) d \xi$ as function of $d / \lambda_{T}$ in Figs. 2, 4. These sums appear in Eq. (7) in the retarded limit where the tensor $\alpha$ is frequency independent. At large temperature all coefficients show a linear temperature dependence, reproducing the classical result of Eq. 13. At small $T$ the coefficient display only a weak temperature dependence which is consistent with the powers of $T$ in Eqs. (9)-(12). An exceptionally strong temperature dependence is observed for the coefficient

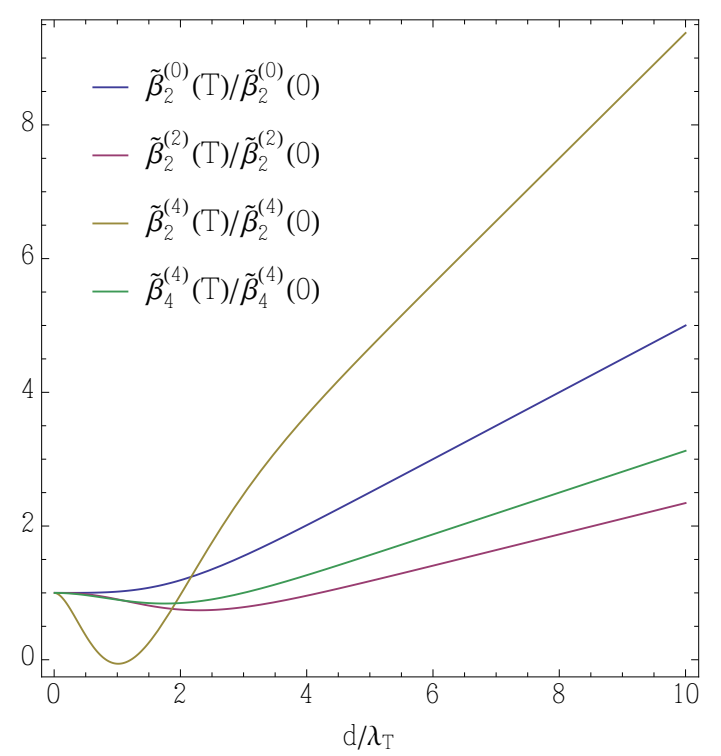

FIG. 3: Same as Fig. 2 but for the coefficients of $\alpha_{z z}^{0}$ in Eq. (7).

$\beta_{2}^{(4)}$, see Fig. 3.

\section{SUMMARY AND IMPLICATIONS}

We have studied the combined effect of thermal fluctuations and surface curvature on the Casimir-Polder interaction at small particle-surface separations. Explicit results for the interaction potential have been obtained in the retarded limit for a perfectly conducting surface. We have presented analytical results at low and high temperatures, and numerical results at arbitrary temperatures. The employed gradient expansion allows for interesting extensions to dielectric surfaces, and more sophisticated models for the particle, including excited atoms or molecules.

Our work opens the prospect of studying a number a novel phenomena in particle-surface interactions, aris- 


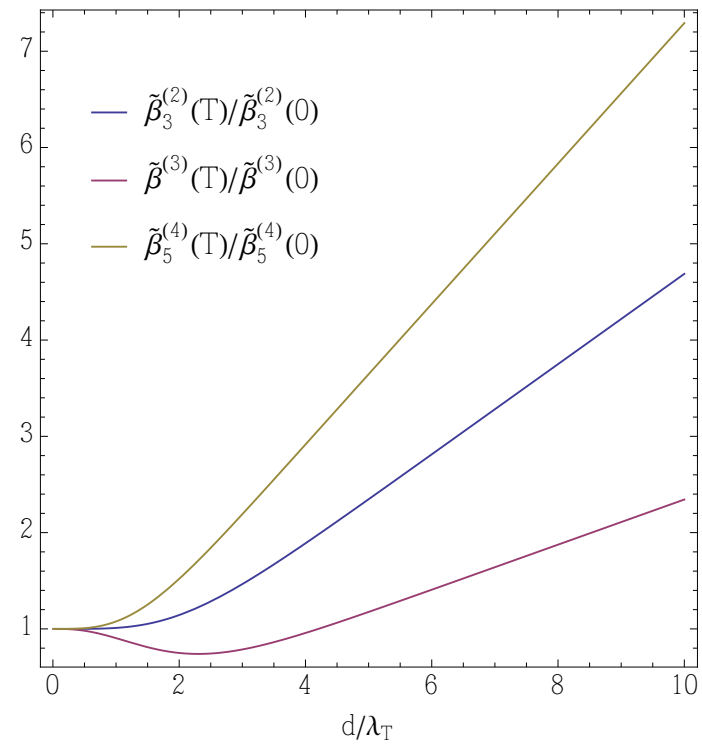

FIG. 4: Same as Fig. 2 but for the coefficients of the mixed components of $\alpha^{0}$ in Eq. (7).

ing from the interplay of thermal excitations and surface shape. It is easy to deduce already from Eq. (7) that curvature of the surface can exert a torque, rotating an anisotropic particle into a specific low energy orientation. The distinct temperature dependencies of the different polarisation components shown in Figs. 2,4 suggest that the preferred orientation at a fixed distance may change upon heating or cooling the system. Non-equilibrium situations, involving an excited (Rydberg) atom or a polar molecule, or a surface held at a different temperature also provide additional avenues for exploration. Recent experiments 24 have demonstrated for planar surfaces a substantial increase of the interaction due to thermally excited surface waves that couple to atomic transitions. The sensitivity of surface-polariton modes to surface curvature suggests the possibility to tailor thermal fluctuations so that they coincide with transitions of the particle, leading to an increased interaction. This idea could be realised by the use of designed nano-structured surfaces.

\section{Acknowledgments}

We thank M. Kardar for valuable discussions.
[1] F. London, Z. Phys. 63, 245 (1930).

[2] H.B.G. Casimir and D. Polder, Phys. Rev. 73, 360 (1948).

[3] H. B. G. Casimir, Proc. K. Ned. Akad. Wet. 51, 793 (1948).

[4] V. A. Parsegian, Van der Waals Forces (Cambridge University Press, 2005).

[5] G.L. Klimchitskaya, U. Mohideen, and V.M. Mostepanenko, Rev. Mod. Phys. 81, 1827 (2009).

[6] Casimir Physics, edited by D.A.R. Dalvit et al., Lecture Notes in Physics Vol. 834 (Springer, New York, 2011).

[7] T. A. Pasquini, M. Saba, G.-B. Jo, Y. Shin, W. Ketterle, D. E. Pritchard, T. A. Savas, and N. Mulders, Phys. Rev. Lett. 97, 093201 (2006).

[8] H. Oberst, D. Kouznetsov, K. Shimizu, J. I. Fujita, and F. Shimizu, Phys. Rev. Lett. 94, 013203 (2005).

[9] B. S. Zhao, S. A. Schulz, S. A. Meek, G. Meijer, and W. Scho llkopf, Phys. Rev. A 78, 010902(R) (2008).

[10] J. D. Perreault, A. D. Cronin, and T. A. Savas, Phys. Rev. A 71, 053612 (2005); V. P. A. Lonij, W. F. Holmgren, and A. D. Cronin, ibid. 80, 062904 (2009).

[11] B. Dobrich, M. DeKieviet, and H. Gies, Phys. Rev. D 78, 125022 (2008).

[12] Ana M. Contreras-Reyes, R. Guerout, P. A. Maia Neto, D.A.R. Dalvit, A. Lamrecht, and S. Reynaud, Phys. Rev. A 82, 052517 (2010).

[13] V.B. Bezerra, E.R. Bezerra de Mello, G.L. Klimchitskaya, V.M. Mostepanenko, and A.A. Saharian, Eur. Phys. J. C 71, 1614 (2011).

[14] P. Rodriguez-Lopez, T. Emig, E. Noruzifar, and R. Zandi, Effect of curvature on the Casimir-Polder interaction, arXiv:1410.0513.

[15] R. Messina, D.A.R. Dalvit, P. A. Maia Neto, A. Lam- brecht, and S. Reynaud, Phys. Rev. A 80, 022119 (2009).

[16] B. V. Derjaguin and I.I. Abrikosova, Sov. Phys. JETP 3, 819 (1957); B. V. Derjaguin, Sci. Am. 203, 47 (1960).

[17] G. Bimonte, T. Emig, and M. Kardar, Phys. Rev. D 90, 081702(R) (2014).

[18] C. D. Fosco, F. C. Lombardo, and F. D. Mazzitelli, Phys. Rev.D 84, 105031 (2011).

[19] G. Bimonte, T. Emig, R. L. Jaffe, and M. Kardar, EPL 97, 50001 (2012).

[20] G. Bimonte, T. Emig, and M. Kardar, Appl. Phys. Lett. 100, 074110 (2012).

[21] V.A. Golyk, M. Kruger, A.P. McCauley, and M. Kardar, EPL 101, 34002 (2013).

[22] C. D. Fosco, F. C. Lombardo, and F. D. Mazzitelli, Phys. Rev. A 88, 062501 (2013).

[23] J. M. Obrecht, R. J. Wild, M. Antezza, L. P. Pitaevskii, S. Stringari, and E. A. Cornell, Phys. Rev. Lett. 98, 063201 (2007).

[24] A. Laliotis, T. Passerat de Silans, I. Maurin, M. Ducloy, and D. Bloch, Nature Comm. $\underline{5}, 4364$ (2014).

[25] B. S. Wu and C. Eberlein, Proc. R. Soc. London A 456, 1931 (2000).

[26] M. Antezza, L. P. Pitaevskii, and S. Stringari, Phys. Rev. A 70, 053619 (2004).

[27] M. Antezza, L. P. Pitaevskii, and S. Stringari, Phys. Rev. Lett. 95, 113202 (2005).

[28] D. M. Harber, J. M. Obrecht, J. M. McGuirk, and E. A. Cornell, Phys. Rev. A 72, 033610 (2005).

[29] T. N. C. Mendes and C. Farina, J. Phys. A 40, 7343 (2007).

[30] S. Y. Buhmann and S. Scheel, Phys. Rev. Lett. 100, 253201 (2008).

[31] V. B. Bezerra, G. L. Klimchitskaya, V. M. Mostepanenko, 
and C. Romero, Phys. Rev. A 78, 042901 (2008).

[32] G. L. Klimchitskaya and V. M. Mostepanenko, J. Phys. A 41, 312002 (2008).

[33] L. P. Pitaevskii, Phys. Rev. Lett. 101, 163202 (2008).

[34] A. Derevianko, B. Obreshkov, and V. A. Dzuba, Phys. Rev. Lett. 103, 133201 (2009).

[35] Z. Zhu and H. Yu, Phys. Rev. A 79, 032902 (2009); 85, 019911 (2012).

[36] J. A. Crosse, S. A. Ellingsen, K. Clements, S. Y. Buhmann, and S. Scheel, Phys. Rev. A 82, 010901 (2010); 82, 029902 (2010).

[37] S. A. Ellingsen, S. Y. Buhmann, and S. Scheel, Phys. Rev. Lett. 104, 223003 (2010).

[38] S. A. Ellingsen, S. Y. Buhmann, and S. Scheel, Phys.
Rev. A 79, 052903 (2009); 84, 060501 (2011).

[39] Z. Zhu, H. Yu, and B. Wang, Phys. Rev. A 86, 052508 (2012).

[40] S. A. Ellingsen, S. Y. Buhmann, and S. Scheel, Phys. Rev. A 85, 022503 (2012).

[41] A. Lambrecht, P. A. Maia Neto, and S. Reynaud, New J. Phys. 8, 243 (2006)

[42] T. Emig, N. Graham, R. L. Jaffe, and M. Kardar, Phys. Rev. Lett. 99, 170403 (2007).

[43] A. Voronovich, Waves Rand. Media, 4, 337 (1994).

[44] We normalize the waves $|\mathbf{k}, Q\rangle$ as in Ref. 43]. Note though that the choice of normalization is irrelevant for the purpose of evaluating the trace in Eq. (1). 